\title{
Robust Parametric Control of Spacecraft Rendezvous
}

\author{
Dake Gu and Yindong Liu \\ School of Automation and Engineering, Northeast Dianli University, No. 169 Changchun Road, Jilin City, Jilin 132012, China \\ Correspondence should be addressed to Dake Gu; dakegu@gmail.com
}

Received 12 December 2013; Accepted 23 April 2014; Published 19 May 2014

Academic Editor: Yuxin Zhao

Copyright (C) 2014 D. Gu and Y. Liu. This is an open access article distributed under the Creative Commons Attribution License, which permits unrestricted use, distribution, and reproduction in any medium, provided the original work is properly cited.

\begin{abstract}
This paper proposes a method to design the robust parametric control for autonomous rendezvous of spacecrafts with the inertial information with uncertainty. We consider model uncertainty of traditional C-W equation to formulate the dynamic model of the relative motion. Based on eigenstructure assignment and model reference theory, a concise control law for spacecraft rendezvous is proposed which could be fixed through solving an optimization problem. The cost function considers the stabilization of the system and other performances. Simulation results illustrate the robustness and effectiveness of the proposed control.
\end{abstract}

\section{Introduction}

With further exploration into the space, a set of complex missions is in the space development agenda such as large-scale structure assembling, sending and picking up astronauts, and repairing, saving, and docking, orbital propellant resupply based on the autonomous rendezvous technology [1]. Due to the essential position, many scholars have been focusing on the control problem during rendezvous and some results enlightened deeper research. In the approximately circular orbit, C-W equations [2], derived by Clohessy and Wiltshire, have been widely applied for the depiction of the relative motion between neighboring spacecrafts. The early stage of control design based on C-W equation revealed a number of open-loop methods such as V-bar, R-bar, dual impulsive, and multiple impulsive [3]. With the benefits of control theory flourishing, plenty of advanced control methods are used to solve the rendezvous problems such as using artificial potential function in [4], sliding mode control in [5], adaptive control in [6], and $\mathrm{H}$-infinity theory in [7].

Though the C-W equation supplies an explicit description of the relative motion for spacecrafts, there is an obstacle when applied in reality that the real-time angle velocity of the target spacecraft could not be obtained accurately as result of detection errors and perturbation from environment. This parameter uncertainty affects the control force and system stability directly. It is necessary to investigate the uncertain model for spacecraft rendezvous not depending on accurate value of real-time angle velocity. The traditional robust control method could deal with parametric uncertainty to recognize rendezvous but some expecting system characters are hard to be included during the control design.

In this paper, the spacecraft rendezvous problem with uncertain parameter would be solved by robust parametric method which allows freedom to improve system performance. The robust control integrates eigenstructure assignment and model reference theory to propose a concise control law for spacecraft rendezvous which takes into consideration the system performance such as the control constraints and fuel saving. In the rest of this paper, a relative motion model with uncertainty for the spacecraft rendezvous is to be established; the design of the robust parametric control law follows; besides, we apply the robust parametric control for an example to illustrate the effectiveness of this design approach.

\section{Problem Formation}

2.1. Equations of Motion. The coordinate frame for the two spacecrafts rendezvous is based on the target spacecraft orbit, described in Figure 1. We set the original point at the target's mass center; $x, y$, and $z$ indicate along-track, the radial, and out of plane components of the position vector of the chaser satellite in the target satellite's local-vertical-local-horizontal (LVLH) frame, respectively. 


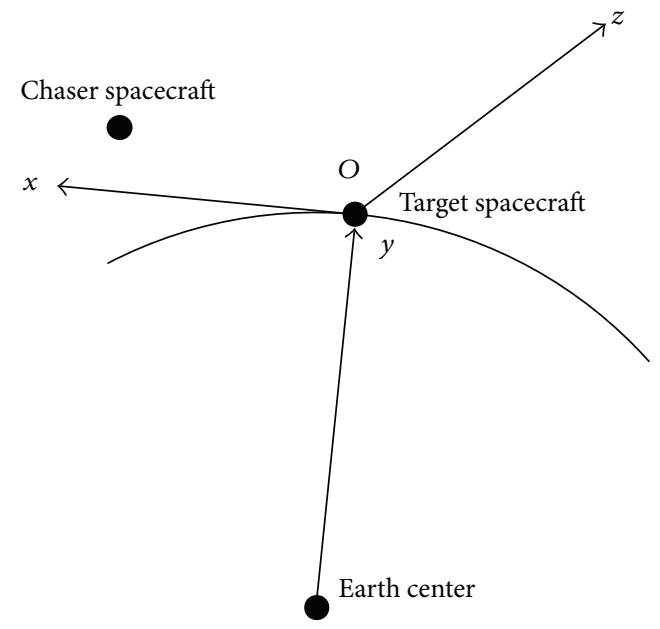

FIGURE 1: Orbital coordinate.

The spacecraft rendezvous in the circle orbit would obey the $\mathrm{C}-\mathrm{W}$ equations

$$
\begin{gathered}
\ddot{x}-2 \omega \dot{y}=-f_{x} \\
\ddot{y}-2 \omega \dot{x}-3 \omega^{2}=-f_{y} \\
\ddot{z}+\omega^{2} z=-f_{z},
\end{gathered}
$$

where $x, y$, and $z$ stand for the relative position between the chase spacecraft and the target spacecraft; $\omega$ represents the average angle velocity of the target spacecraft; $f_{x}, f_{y}$, and $f_{z}$ stand for the control acceleration on each axis.

According to the equation, the state and control vector can be described as

$$
\begin{aligned}
& X=\left[\begin{array}{llllll}
x & y & z & \dot{x} & \dot{y} & \dot{z}
\end{array}\right]^{T}, \\
& u=\left[\begin{array}{llll}
-f_{x} & -f_{y} & -f_{z}
\end{array}\right]^{T},
\end{aligned}
$$

and output vector $Y$ can be

$$
Y=\left[\begin{array}{lll}
x & y & z
\end{array}\right]^{T} \text {. }
$$

Then, we get

$$
\begin{gathered}
\dot{X}=A X+B u \\
Y=C X,
\end{gathered}
$$

where

$$
\begin{gathered}
A=\left[\begin{array}{cccccc} 
& \mathbf{0}_{3} & & & \mathbf{I}_{3} & \\
0 & 0 & 0 & 0 & -2 \omega & 0 \\
0 & 3 \omega^{2} & 0 & 2 \omega & 0 & 0 \\
0 & 0 & -\omega^{2} & 0 & 0 & 0
\end{array}\right] \\
B=\left[\begin{array}{l}
\mathbf{0}_{3} \\
\mathbf{I}_{3}
\end{array}\right], \quad C=\left[\begin{array}{ll}
\mathbf{I}_{3} & \mathbf{0}_{3}
\end{array}\right],
\end{gathered}
$$

and $\mathbf{0}_{3}$ represents the matrix with the values of all elements equal to zero; $\mathbf{I}_{3}$ represents the unit matrix.
2.2. Problem Description. The classical C-W equations need accurate angle velocity simultaneously which is difficult to obtain due to the detection error. Therefore, we consider the uncertain item $\theta$ to the angle velocity to make the system model closer to reality.

When the angle velocity changes are

$$
\omega=\omega_{0}(1+\theta)
$$

the system model can be described as

$$
\begin{gathered}
\dot{X}=A_{c} X+B u \\
Y=C X
\end{gathered}
$$

where

$$
\begin{gathered}
A_{c}=A_{0}+\Delta A \\
A_{0}=\left[\begin{array}{cccccc} 
& \mathbf{0}_{3} & & \mathbf{I}_{3} & \\
0 & 0 & 0 & 0 & -2 \omega_{0} & 0 \\
0 & 3 \omega_{0}^{2} & 0 & 2 \omega_{0} & 0 & 0 \\
0 & 0 & -\omega_{0}^{2} & 0 & 0 & 0
\end{array}\right] \\
\Delta A=\left[\begin{array}{cccccc}
0 \\
0 & \mathbf{0}_{3} & 0 & 0 & -2 \theta \omega_{0} & 0 \\
0 & 3 \omega_{0}^{2}\left(2 \theta+\theta^{2}\right) & 0 & 2 \theta \omega_{0} & 0 & 0 \\
0 & 0 & -\omega_{0}^{2}\left(2 \theta+\theta^{2}\right) & 0 & 0 & 0
\end{array}\right] .
\end{gathered}
$$

The object of the designing control law is to recognize

$$
\lim _{t \rightarrow \infty}\left[Y(t)-Y_{r}(t)\right]=0,
$$

where $Y(t)$ is the output of the system and $Y_{r}(t)$ represent the reference relative position between chase spacecraft and target spacecraft. Meanwhile, the uncertainty brings trouble to the stability of the system which would be taken into consideration during designing the control law.

\section{Design of Robust Parametric Control}

The design of the control law aims at reaching the reference point of the chase spacecraft and keeping the closed loop system stable. It could be separated into two parts as stabilization controller and trajectory tracking controller.

3.1. Trajectory Tracking Controller. To begin with, we would design the tracking controller based on the model reference theory. Lemma 1 supplies theoretical evidence for the linear tracking problems referred to [8].

Lemma 1. For the system, if the stabilization feedback control law $K$ exists, the control law following the form as

$$
u=K X+G Y_{r}
$$

would obtain the result of tracking reference signal, which means that

$$
\lim _{t \rightarrow \infty}\left[Y(t)-Y_{r}(t)\right]=0
$$


where feedforward control law G could be calculated from the following equation:

$$
G=U-K Z
$$

and $U, Z$ could be calculated as

$$
\left[\begin{array}{l}
Z \\
U
\end{array}\right]=\left[\begin{array}{ll}
A & B \\
C & D
\end{array}\right]^{-1}\left[\begin{array}{l}
0 \\
I
\end{array}\right] .
$$

According to Lemma 1, the rendezvous system could track the reference position when the feedback control law $K$ stabilizes the system. Then, the critical task of designed controller is to find a robust stabilization control law $K$. Regarding the eigenstructure assignment of linear system, some useful results would be utilized in the later part which are from [8].

Lemma 2. Suppose $A \in \mathbb{R}^{n \times n}, B \in \mathbb{R}^{n \times r}$, and $(A, B)$ is controllable. $s_{i}, i=1,2, \ldots, n$, are a set of complex numbers, which are symmetric about the real axis. Then, the matrices $K \in \mathbb{R}^{r \times n}$ and $V \in \mathbb{C}^{n \times n}$ satisfying

$$
\bar{A}=A+B K=V \operatorname{diag}\left(s_{1}, s_{2}, \ldots, s_{n}\right) V^{-1}
$$

are given by

$$
\begin{gathered}
K=W V^{-1} \\
V=\left[\begin{array}{llll}
v_{1} & v_{2} & \cdots & v_{n}
\end{array}\right], \quad v_{i}=N\left(s_{i}\right) f_{i} \\
w=\left[\begin{array}{llll}
w_{1} & w_{2} & \cdots & w_{n}
\end{array}\right], \quad w_{i}=D\left(s_{i}\right) f_{i},
\end{gathered}
$$

where $f_{i} \in \mathbb{C}^{r}, i=1,2, \ldots, n$, are arbitrary vectors which satisfy

$$
\begin{gathered}
f_{i}=\bar{f}_{j} \quad \text { if } s_{i}=\bar{s}_{j} \\
\operatorname{det}(V) \neq 0
\end{gathered}
$$

and $N(s)$ and $D(s)$ are right comprime polynomial matrices satisfying

$$
(s I-A)^{-1} B=N(s) D^{-1}(s) .
$$

For the rendezvous system in this paper, we could calculate according to Lemma 2 as

$$
\begin{gathered}
N(s)=\left[\begin{array}{cccccc}
-1 & 0 & 0 & -s & 0 & 0 \\
0 & -1 & 0 & 0 & -s & 0 \\
0 & 0 & -1 & 0 & 0 & -s
\end{array}\right]^{T} \\
D(s)=\left[\begin{array}{ccc}
-s^{2} & -2 \omega_{0} s & 0 \\
-2 \omega_{0} s & 3 \omega_{0}^{2}-s^{2} & 0 \\
0 & 0 & \omega_{0}^{2}-s^{2}
\end{array}\right] .
\end{gathered}
$$

Lemma 2 supplies a concise parametric formula for state feedback law $K$ in which the poles of the closed-loop system are included. Proper poles would not only guarantee the system stabilization but also enhance system characters through optimization in some specific fields. Besides, the parametric method offers all kinds of freedom to design the control system with the free parametric vectors $f_{i}, i=$ $1,2, \ldots, n$, which enable us to adjust these parameters for system stabilization.
3.2. Stabilization Controller. Using the control law

$$
u=K X+G Y_{r}
$$

the closed-loop system can be described as

$$
\dot{X}=\left(A_{k}+\Delta A\right) X+B G Y_{r}
$$

where

$$
A_{k}=A_{0}+B K
$$

When $A_{k}$ is a nondefective matrix and the closed-loop system owns the required poles $s_{i}(i=1,2, \ldots, n)$, the sufficient condition for the system stabilization with the uncertainty item $\Delta A$ is [9]

$$
\|\Delta A\|_{2}<\frac{1}{\|P\|_{2}}
$$

where $P$ is a symmetric positive definite solution of the following:

$$
A_{k}^{T} P+P A_{k}=-2 I .
$$

Lemma 3 provides the parametric expression for $P$ based on the eigenstucture of the system.

Lemma 3. The solution to (23) has the following parametric representation:

$$
P=2 V^{-T} Q V^{-1}
$$

where

$$
Q=\left[-\frac{v_{i}^{T} v_{j}}{s_{i}+s_{j}}\right]_{n \times n}
$$

and $s_{i}, v_{i}, i=1,2, \ldots, n$, are respectively the eigenvalues and corresponding eigenvectors of $A_{k}$.

For a better stabilization system, we hope to minimize $\|P\|_{2}$ which is the result of some adjustments for the parameters $s_{i}(i=1,2, \ldots, n)$ and $f_{i}(i=1,2, \ldots, n)$.

3.3. Optimization of Control Law. We have established the connection between the system characters and the parameters $s_{i}$ and $f_{i}$ through the design of the control law. Therefore, the design problem for the rendezvous system can be converted into the following nonlinear optimization problem:

$$
\begin{array}{ll}
\min & J\left(s_{i}, f_{i}\right) \\
\text { s.t. } & a_{i} \leq \operatorname{Re}\left(s_{i}\right) \leq b_{i}<0 \\
& c_{i} \leq \operatorname{Im}\left(s_{i}\right) \leq d_{i}, \quad i=1,2, \ldots, n,
\end{array}
$$

where $a_{i}, b_{i}, c_{i}$, and $d_{i}$ specify the desired areas of the closedloop eigenvalues. 


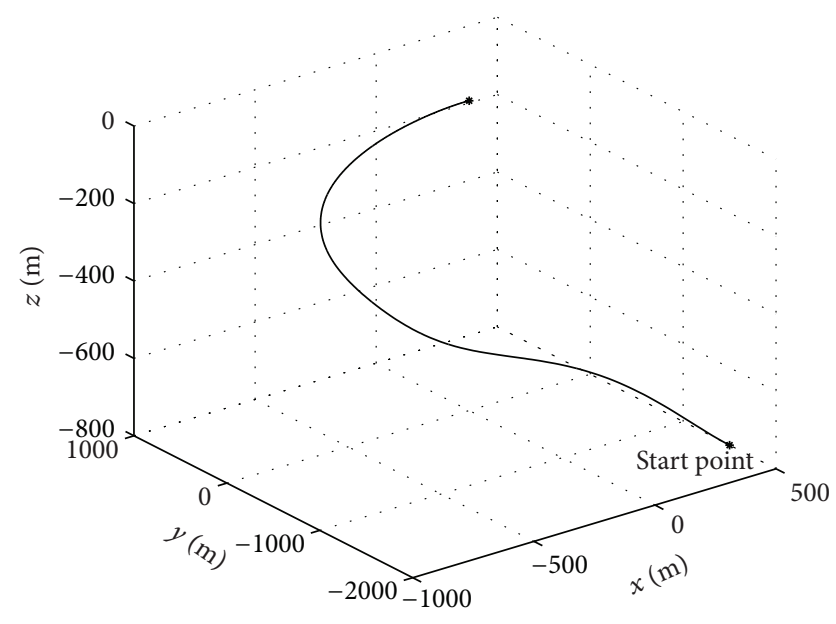

Figure 2: Autonomous rendezvous trajectory of chaser.
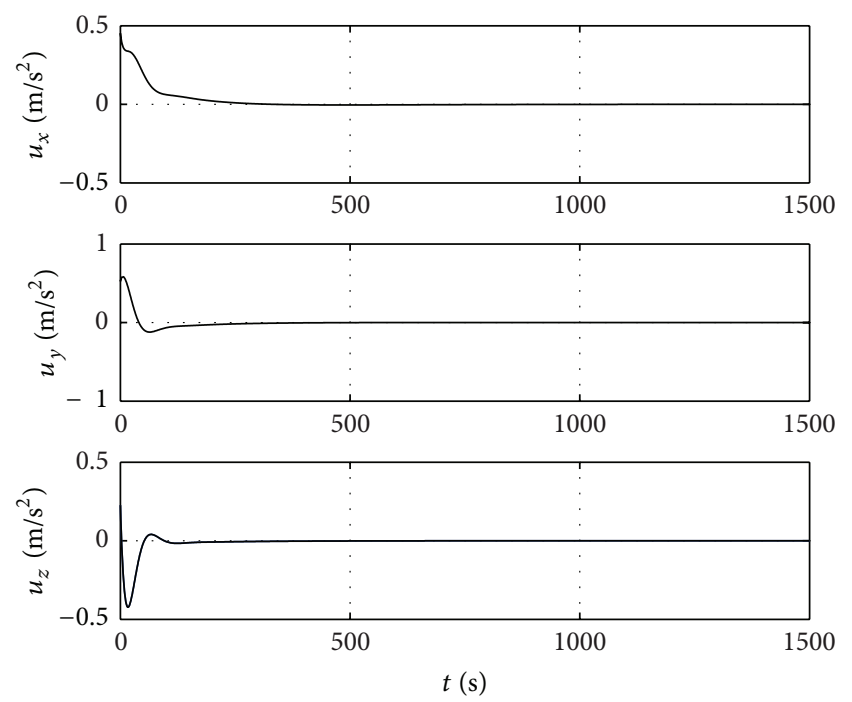

FIGURE 3: Control inputs during rendezvous mission.

The performance index is chosen as follows:

$$
J=\alpha L^{T} K^{T} K L+\beta\|K\|_{F}+\gamma\|P\|_{2},
$$

where $L$ denotes the initial state of system; $\alpha, \beta$, and $\gamma$ are the weighting factors. The first part of (27) is chosen due to the consideration of the input constraint. The second item of (27) takes into consideration fuel consumption. The last part of (27) is used for global stability of the rendezvous system.

The optimization discussed above could be solved resorting to the optimization tool in MATLAB for its convenience. Then, the poles $s_{i}(i=1,2, \ldots, n)$ of the system and free parametric vectors $f_{i}(i=1,2, \ldots, n)$ would be fixed to calculate the feedback matrix for the robust control.

\section{Numerical Simulations}

In this section, our control law designed through the method proposed above would be tested by an example of spacecrafts in the final approaching in rendezvous mission. With the assumption that the target is in the geosynchronous orbit,
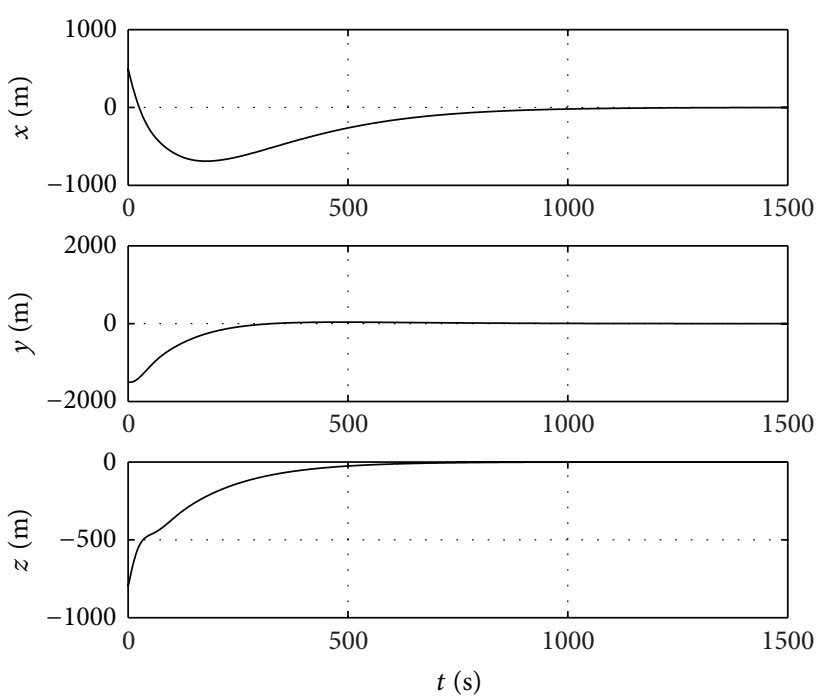

FIgURE 4: Relative position of two spacecraft.

we set the standard angle velocity $\omega_{0}=7.2921 \times 10^{-5} \mathrm{rad} / \mathrm{s}$. Suppose the initial state vector is

$$
X(0)=\left[\begin{array}{llllll}
500 & -1500 & -800 & -1.0 & 0.1 & 0.1
\end{array}\right]^{T}
$$

and the desired final state is

$$
X\left(t_{f}\right)=\left[\begin{array}{llllll}
0 & 0 & 0 & 0 & 0 & 0
\end{array}\right]^{T},
$$

where $t_{f}$ is final time of the rendezvous mission. The weighting factors in the performance index $J$ are

$$
\alpha=0.1, \quad \beta=15, \quad \gamma=0.0001 .
$$

Specify the desired closed-loop eigenvalue regions as

$$
-0.1 \leq \operatorname{Re}\left(s_{i}\right) \leq-0.001, \quad-0.1 \leq \operatorname{Im}\left(s_{i}\right) \leq 0.1 .
$$

By solving the optimization problem (26a), (26b), and (26c), we gain the poles $s_{i}$ of the system and free parametric vectors $f_{i}, i=1,2, \ldots, 6$,

$$
\begin{array}{rl}
s_{1,2}=-0.0064 \mp 0 & 0.0008 \mathrm{i}, \quad s_{3,4}=-0.0456 \mp 0.0519 \mathrm{i}, \\
s_{5,6}=-0.0606 \mp 0.0094 \mathrm{i} \\
f_{1,2}=\left[\begin{array}{c}
-11.0091 \mp 50.3604 \mathrm{i} \\
-0.8446 \pm 20.1762 \mathrm{i} \\
-2.1281 \pm 0.5444 \mathrm{i}
\end{array}\right], \\
f_{3,4}=\left[\begin{array}{c}
14.0820 \pm 2.7142 \mathrm{i} \\
5.5407 \mp 20.7854 \mathrm{i} \\
-12.6640 \pm 26.1508 \mathrm{i}
\end{array}\right], \\
f_{5,6}=\left[\begin{array}{c}
31.5687 \pm 7.1917 \mathrm{i} \\
-1.5892 \pm 4.2163 \mathrm{i} \\
-0.3478 \pm 12.9690 \mathrm{i}
\end{array}\right] .
\end{array}
$$

According to Lemma 2 and (15), we get 


$$
\begin{gathered}
V=\left[\begin{array}{cccccc}
11.0091+50.3604 \mathrm{i} & 11.0091-50.3604 \mathrm{i} & -14.0820-2.7142 \mathrm{i} & -14.0820+2.7142 \mathrm{i} & -31.5687-7.1917 \mathrm{i} & -31.5687+7.1917 \mathrm{i} \\
0.8446-20.1762 \mathrm{i} & 0.8446+20.1762 \mathrm{i} & -5.5407+20.7854 \mathrm{i} & -5.5407-20.7854 \mathrm{i} & 1.5892-4.2163 \mathrm{i} & 1.5892+4.2163 \mathrm{i} \\
2.1281-0.5444 \mathrm{i} & 2.1281+0.5444 \mathrm{i} & 12.6640-26.1508 \mathrm{i} & 12.6640+26.1508 \mathrm{i} & 0.3478-12.9690 \mathrm{i} & 0.3478+12.9690 \mathrm{i} \\
-0.1107-0.3135 \mathrm{i} & -0.1107+0.3135 \mathrm{i} & 0.7830-0.6071 \mathrm{i} & 0.7830+0.6071 \mathrm{i} & 1.9807+0.1391 \mathrm{i} & 1.9807-0.1391 \mathrm{i} \\
0.0107+0.1298 \mathrm{i} & 0.0107-0.1298 \mathrm{i} & -0.8261-1.2354 \mathrm{i} & -0.8261+1.2354 \mathrm{i} & -0.056+0.2704 \mathrm{i} & -0.056-0.2704 \mathrm{i} \\
-0.0132+0.0052 \mathrm{i} & -0.0132-0.0052 \mathrm{i} & 0.7797+1.8497 \mathrm{i} & 0.7797-1.8497 \mathrm{i} & 0.1008+0.7892 \mathrm{i} & 0.1008-0.7892 \mathrm{i}
\end{array}\right] \\
W=\left[\begin{array}{cccccc}
-0.0001+0.0022 \mathrm{i} & -0.0001-0.0022 \mathrm{i} & 0.0217-0.0651 \mathrm{i} & 0.0217+0.0651 \mathrm{i} & -0.1050-0.0617 \mathrm{i} & -0.1050+0.0617 \mathrm{i} \\
0.0002-0.0009 \mathrm{i} & 0.0002+0.0009 \mathrm{i} & -0.0949-0.0389 \mathrm{i} & -0.0949+0.0389 \mathrm{i} & 0.0108-0.0132 \mathrm{i} & 0.0108+0.0132 \mathrm{i} \\
0.0001 & 0.0001 & 0.1160+0.0760 \mathrm{i} & 0.1160-0.0760 \mathrm{i} & 0.0160+0.0461 \mathrm{i} & 0.0160-0.0461 \mathrm{i}
\end{array}\right]
\end{gathered}
$$

Then, the stabilization control law $K$ to (15) is

$$
K=\left[\begin{array}{cccccc}
-0.0011 & -0.0020 & 0.0027 & -0.0685 & -0.0435 & -0.0271 \\
0.0005 & 0.0013 & -0.0028 & 0.0170 & 0.0149 & -0.0587 \\
0.0000 & -0.0009 & 0.0013 & 0.0053 & -0.1128 & -0.0040
\end{array}\right]
$$

Assume the uncertainty $\theta=0.01$ which leads to

$$
\|\Delta A\|_{2}=1.4584 \times 10^{-6} .
$$

Meanwhile, the closed-loop poles $s_{i}$ and parametric vectors $f_{i}$ could be used to calculate as

$$
\frac{1}{\|P\|_{2}}=1.5037 \times 10^{-5} \text {. }
$$

It is obvious that the rendezvous process could reach the desired state with the control law $K$ when the inequality (22) has satisfied. The rendezvous trajectory and the relative position of the two spacecraft are showed in Figures 2 and 4 and the effectiveness could be proved simultaneously. Due to the proper optimization function, the control inputs have been constrained to $[-1,1]$ which can be seen in Figure 3 . The motion in every axis direction changes smoothly so that the simulation system gets closer to the real engineering requirement showed in Figure 3.

\section{Conclusion}

This paper has proposed a method to design the robust control law for spacecraft rendezvous in the final approach subject to parameter uncertainty in near circle orbit. Based on the eigenstructure assignment and model reference theory, the control law is constructed with the closed-loop poles and design freedom. Through solving an optimization problem, we obtain the poles and parametric vectors to calculate the control law which has been proved useful by simulation.

\section{Conflict of Interests}

The authors declare that there is no conflict of interests regarding the publication of this paper.

\section{Acknowledgments}

This work was supported by National Natural Science Foundation of China (no. 61074111), Natural Science Foundation of Jilin Province, China (no. 20140520061JH), and Educational Commission Foundation of Jilin Province, China (no. 2013122).

\section{References}

[1] N. F. Dipprey and S. J. Rotenberger, "Orbital express propellant resupply servicing," in Proceedings of the 39th AIAA/ASME/SAE/ASEE Joint Propulsion Conference and Exhibit, pp. 20-23, Huntsville, Ala, USA, July 2003.

[2] W. H. Clohessy and R. S. Wiltshire, "Terminal guidance system for satellite rendezvous," Journal of The Aerospace Science, vol. 27, no. 9, pp. 653-658, 1960.

[3] H. B. Hablani, M. L. Tapper, and D. J. Dana-Bashian, "Guidance and relative navigation for autonomous rendezvous in a circular orbit," Journal of Guidance, Control, and Dynamics, vol. 25, no. 3, pp. 553-562, 2002.

[4] I. Lopez and C. R. McInnes, "Autonomous rendezvous using artificial potential function guidance," Journal of Guidance, Control, and Dynamics, vol. 18, no. 2, pp. 237-241, 1995.

[5] X. Zhao, Y.X. Jiang, and Y.J. Wu, "Multi-slide-mode control for flying-around of rendezvous and docking," Journal of Astronautics, vol. 31, no. 1, pp. 143-147, 2010.

[6] P. Singla, K. Subbarao, and J. L. Junkins, "Adaptive output feedback control for spacecraft rendezvous and docking under measurement uncertainty," Journal of Guidance, Control, and Dynamics, vol. 29, no. 4, pp. 892-902, 2006.

[7] H. Gao, X. Yang, and P. Shi, "Multi-objective robust $H_{\infty}$ control of spacecraft rendezvous," IEEE Transactions on Control Systems Technology, vol. 17, no. 4, pp. 794-802, 2009.

[8] G.R. Duan, "Solutions of the equation $A V+B W=V F$ and their application to eigenstructure assignment in linear systems," IEEE Transactions on Automatic Control, vol. 38, no. 2, pp. 276280, 1993.

[9] G. R. Duan, G. W. Irwin, and G. P. Liu, "Disturbance attenuation in linear systems via dynamical compensators: a parametric eigenstructure assignment approach," IEE Proceedings: Control Theory and Applications, vol. 147, no. 2, pp. 129-136, 2000. 


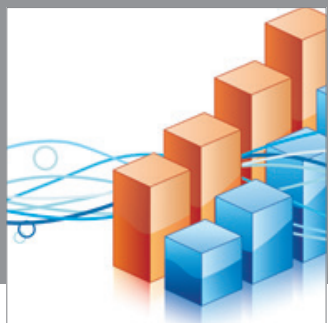

Advances in

Operations Research

mansans

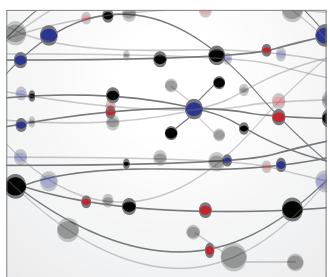

The Scientific World Journal
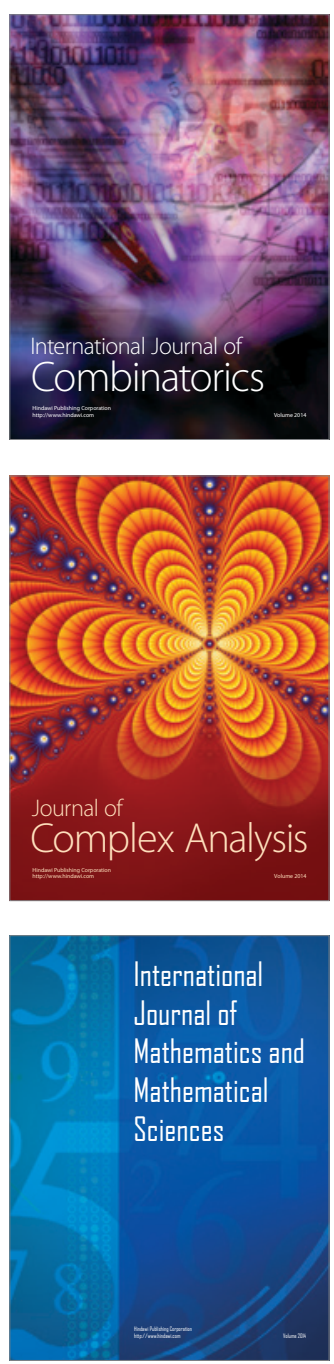
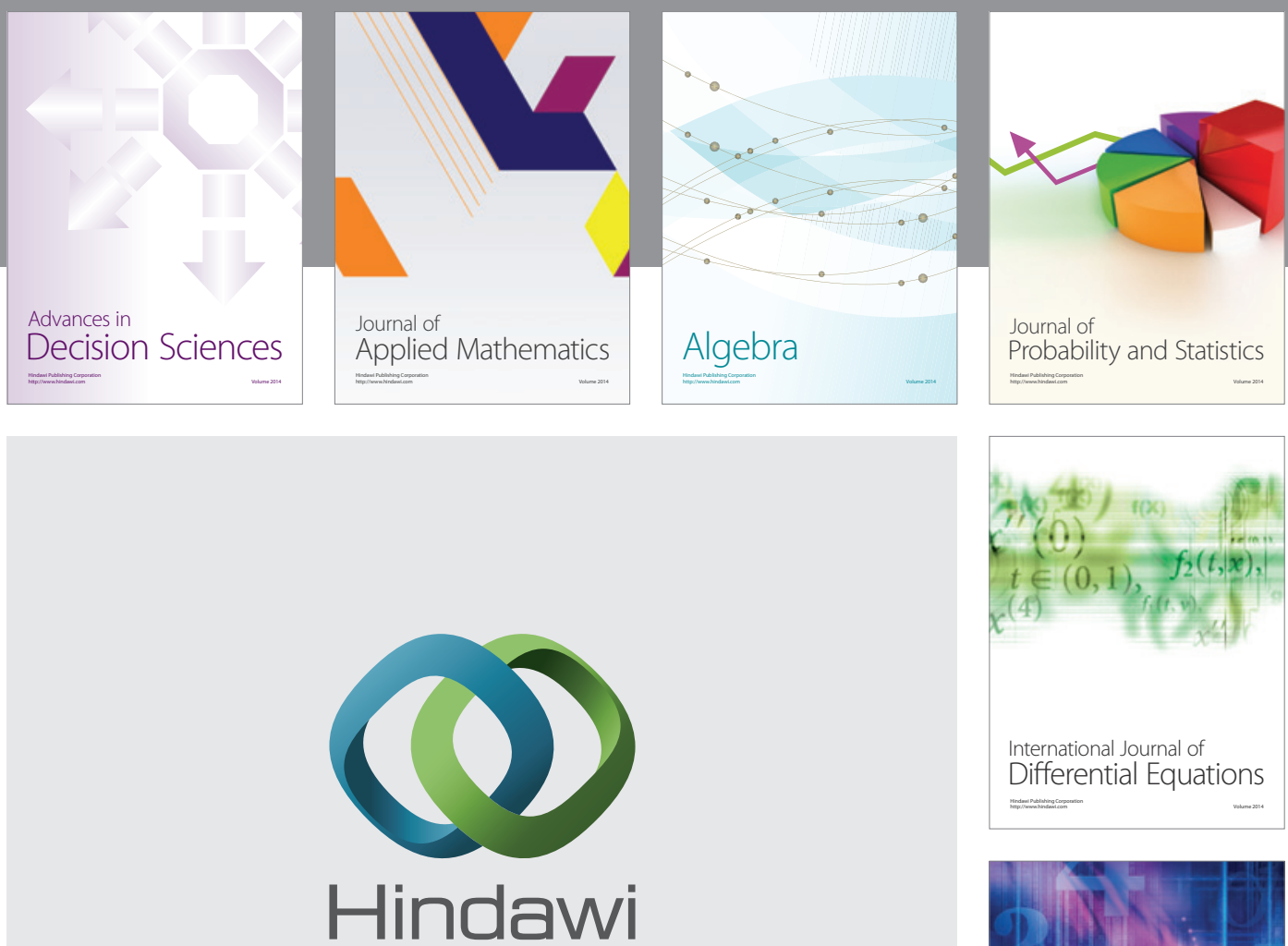

Submit your manuscripts at http://www.hindawi.com
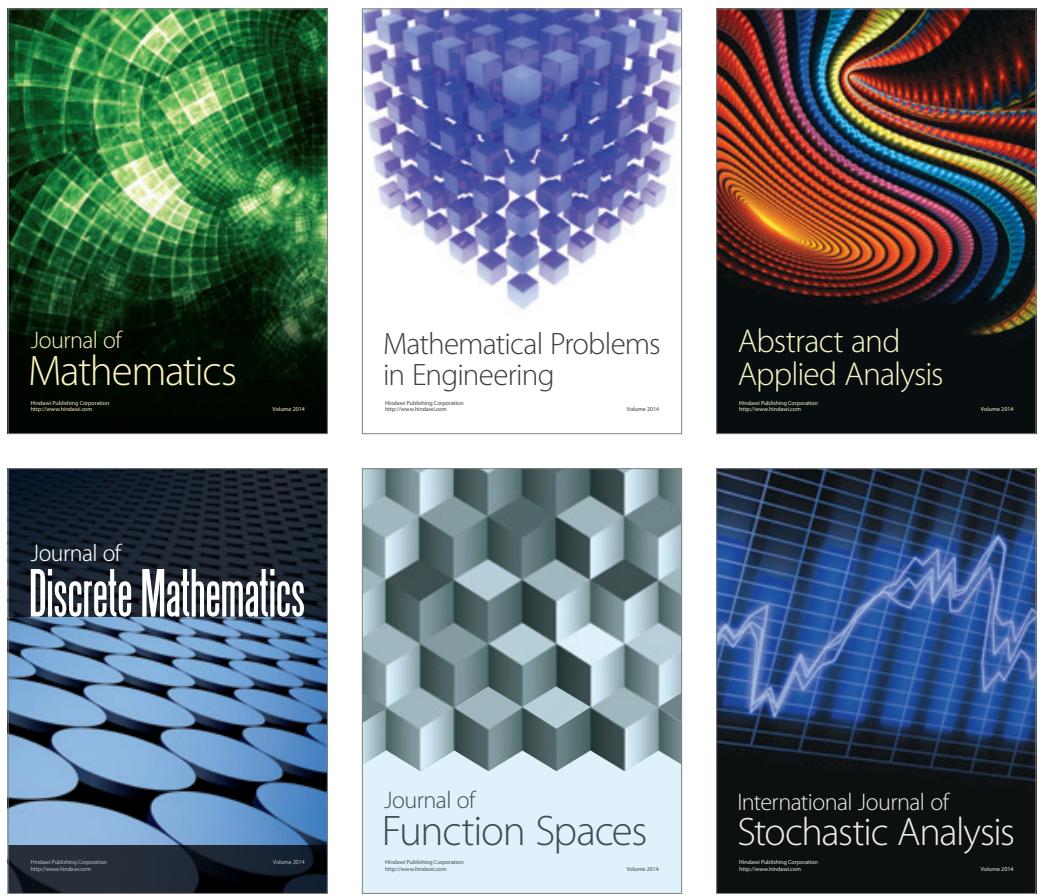

Journal of

Function Spaces

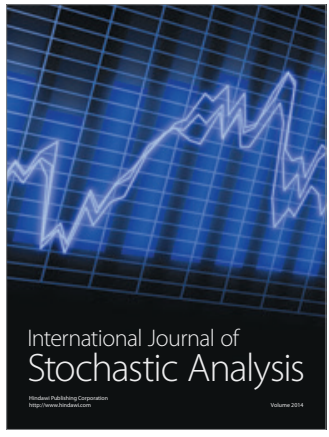

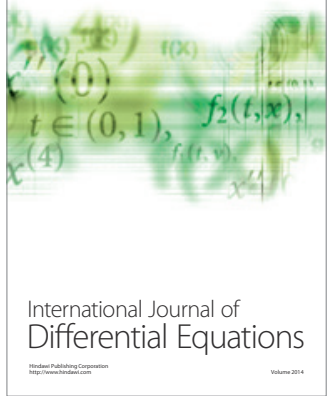
